\title{
Aislamiento e identificación de especies pertenecientes a los géneros Aeromonas, Vibrio y Plesiomonas procedentes de muestras extra-intestinales en Cuba
}

\author{
Luis E. Cabrera R., Graciela Castro E., M. Margarita Ramírez A., Alina Llop H., Rafael Llanes C., \\ Neisideismy Castañeda E., Anabel Fernández A. y Laura Bravo F.
}

Centro Municipal de Higiene y Epidemiología de Güines (LECR) Instituto Politécnico Nacional.

México Becario COFAA, EDD Escuela Nacional de Ciencias Biológicas

Laboratorio de Bacteriología Médica (GCE) Instituto de Medicina Tropical Pedro Kourí, La Habana, Cuba (MMRA, ALH, RLC, NCE, AFA, LBF)

Recibido: 1 noviembre 2006 Aceptado: 7 marzo 2007

Correspondencia a: Laura Bravo Fariñas laura@ipk.sld.cu

\author{
Isolation and identification of species from the genera Aeromonas, Vibrio, \\ and Plesiomonas from extraintestinal samples in Cuba
}

Extraintestinal infections caused by the genera Aeromonas, Vibrio and Plesiomonas have high morbidity and mortality rates in different areas of world. From January 2002 to December 2003, the National Reference Laboratory for Acute Diarrhoeal Diseases of the Pedro Kourí Tropical Medicine Institute received 95 gramnegative, facultative anaerobic, oxidase positive bacilli strains from different extraintestinal specimen (blood, ear exudates, infected wounds, conjunctive exudates, urine, and catheters, among others) sent by different provincial laboratories along the country. Aeromonas caviae, Aeromonas veronii bv sobria, Aeromonas jandaei, Vibrio cholerae no-O1, Vibrio vulnificus, Vibrio fluvialis and Plesiomonas shigelloides were the species most frequently found in the sample analysed.

Key words: Extraintestinal infections; Aeromonas; Vibrio; Plesiomonas.

Palabras claves: Infección extra-intestinal; Aeromonas; Vibrio; Plesiomonas.

\section{Introducción}

$\mathbf{L}$ os bacilos gramnegativos, anaerobios facultativos, oxidasa positiva, pertenecen a los géneros Aeromonas, Vibrio y Plesiomonas, ubicados taxonómicamente en las familias Aeromonadaceae, Vibrionaceae y Enterobacteriaceae, respectivamente ${ }^{1}$.

Es reconocido el incremento de las infecciones por los microorganismos pertenecientes a los mencionados géneros, como agentes etiológicos de procesos infecciosos en órganos y sistemas vitales para el organismo, comprometiendo en ocasiones la vida del paciente ${ }^{2-4}$.

A pesar de los adelantos en el control de las enfermedades transmisibles, cada día son más frecuentes las infecciones intestinales, extra-intestinales y nosocomiales por las especies de los géneros descritos anteriormente, constituyendo una amenaza grave para la salud de la población mundial, por el amplio espec- tro de expresiones clínicas que producen (endocarditis, septicemia, bacteriemia, meningoencefalitis, neumonía, peritonitis e infección hepatobiliar, celulitis, etc) $)^{5-7}$.

Al existir escasos trabajos científicos publicados en Cuba y en el área del Caribe sobre el aislamiento e identificación de los géneros Aeromonas, Vibrio y Plesiomonas en muestras clínicas, nos propusimos profundizar en el diagnóstico microbiológico de estos microorganismos.

\section{Material y Métodos}

Se estudiaron 95 cepas de bacilos gramnegativos, anaerobios facultativos, oxidasa positiva, conservados en el Laboratorio Nacional de Referencia de Enfermedades Diarreicas Agudas del Instituto de Medicina Tropical "Pedro Kourí" (LNR/EDA/IPK), procedentes de diferentes laboratorios de microbiología clínica de 
los Centros Provinciales de Higiene y Epidemiología del país que incluyeron Pinar del Río, Ciudad de la Habana, Matanzas, Cienfuegos, Villa Clara, Camagüey, Las Tunas, Holguín, Granma, Santiago de Cuba, Guantánamo y del Centro Municipal de la Isla de la Juventud, en el período comprendido entre enero del 2002 y diciembre del 2003. Estos microorganismos fueron aislados e identificados de diferentes muestras extra-intestinales de pacientes con signos y síntomas de infección local o generalizada.

Muestras extra-intestinales: Hemocultivos $(n=47)$, exudados óticos $(n=13)$, pus de heridas $(n=8)$, exudados conjuntivales $(\mathrm{n}=5)$, urocultivos $(\mathrm{n}=5)$, de catéter $(\mathrm{n}=4)$, exudados de lesión de piel $(\mathrm{n}=2)$, exudados faríngeos $(\mathrm{n}=2)$, líquido cefalorraquídeo $(\mathrm{n}=1)$, bilicultivo $(n=1)$, secreción articular $(n=1)$, restos placentarios $(n=1)$, esputo $(n=1)$, exudado de úlcera de pie $(n=1)$, exudado de herida quirúrgica $(n=1)$, exudado vaginal $(n=1)$ y exudado endocervical $(n=1)$.

Para el aislamiento de los microorganismos pertenecientes a los géneros, Aeromonas, Vibrio y Plesiomonas las muestras fueron sembradas en medios universales, diferenciales y selectivos. La identificación en géneros y especies se realizó según el flujograma de diagnóstico (Figura 1).

A las cepas que presentaban codificación genética para la enzima citocromo-oxidasa se les realizaron estudios fisiológicos y antigénicos correspondientes para ubicarlas en los géneros, Aeromonas, Vibrio y Plesiomonas $^{1}$. Posteriormente, se aplicaron los siguientes esquemas para la determinación en especies. Las especies del género Aeromonas se determinaron por el Aerokey $11^{8}$. El Aerosquema fue utilizado para identificar especies tanto del género Aeromonas como para el género Plesiomonas shigelloides ${ }^{9}$. Los estudios fisiológicos y antigénicos establecidos en el Manual de Bacteriología Analítica fueron utilizados para la identificación en especie de los aislados pertenecientes al género Vibrio $^{10}$.

\section{Resultados}

De las 95 cepas estudiadas, $85(89,4 \%)$ correspondieron al género Aeromonas, 7 (7,3\%) al género Vibrio y $3(3,3 \%)$ al género Plesiomonas.

Las muestras en que con mayor frecuencia se aislaron los microorganismos de los géneros Aeromonas, Vibrio y Plesiomonas fueron: exudados óticos, pus de heridas, exudado conjuntival, urocultivos y hemocultivo, resultando esta última la muestra extra-intestinal en la cual fueron aislados los 3 géneros en estudio (Tabla 1). Las especies identificadas con mayor frecuencia, en el género Aeromonas, fueron: A. jandei, $A$. veronii bv sobria, $A$. hydrophila y $A$. caviae; en el género Vibrio fueron: $V$. cholerae no - O1, $V$. fluvialis y $V$. vulnificus.

\section{Discusión}

La gastroenteritis aguda es la forma de presentación clínica más frecuente de las infecciones por Aeromonas, Vibrio y Plesiomonas. En las últimas décadas se han publicado numerosos trabajos en los cuales se ha evidenciado un incremento de las infecciones extra-intestinales causadas por los miembros de estos géneros. La detección requiere de una buena historia clínica y el uso apropiado de los procedimientos para el aislamiento e identificación por parte del laboratorio para confirmar estas infecciones ${ }^{11}$.

Los microorganismos pertenecientes a los géneros Aeromonas, Vibrio y Plesiomonas, pueden ser recu-

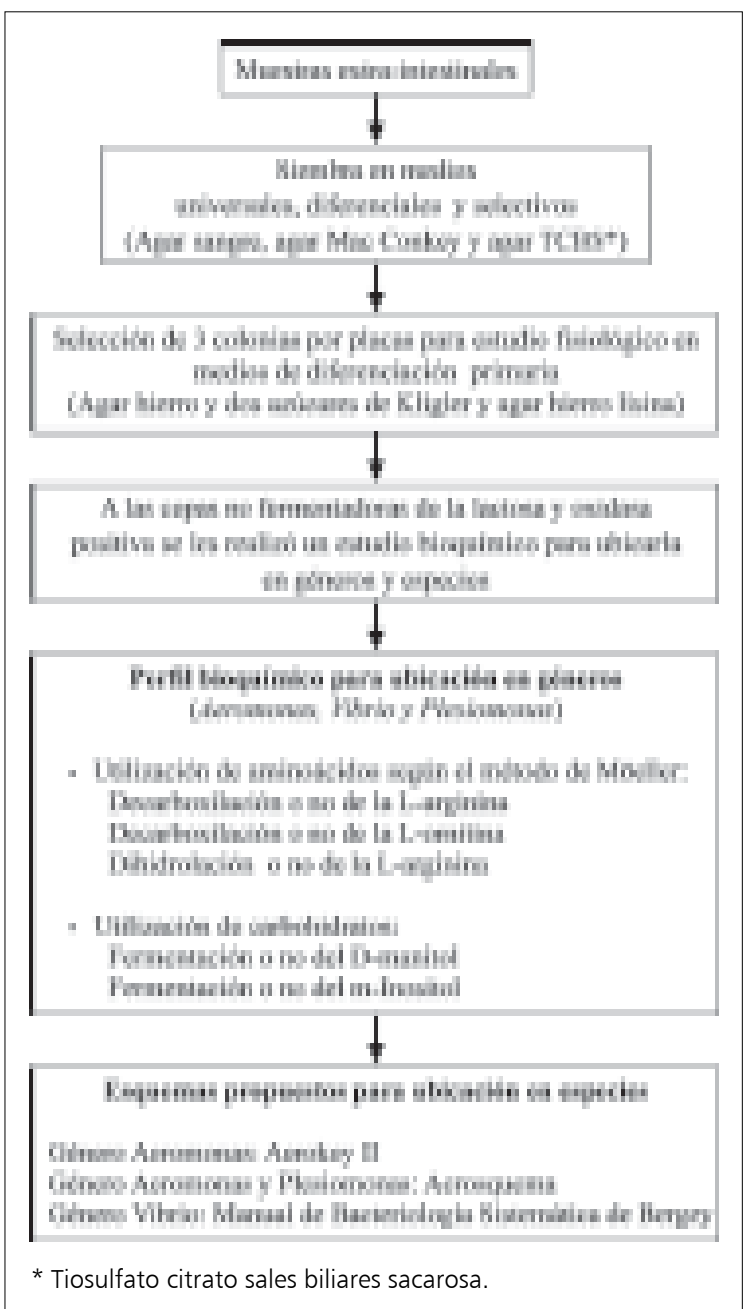

Figura 1. Flujograma de diagnóstico. 


\section{Tabla 1. Distribución de los géneros y especies de las cepas aisladas de muestras extra-intestinales}

\begin{tabular}{|c|c|c|c|}
\hline Muestras clínicas & Aeromonas & Vibrio & Plesiomonas \\
\hline Hemocultivo (47) & $\begin{array}{l}\text { A. jandaei (12) } \\
\text { A. veronii bv sobria (8) } \\
\text { A. trota (6) } \\
\text { A. caviae (5) } \\
\text { A. hydrophila (4) } \\
\text { Aeromonas sp (10) }\end{array}$ & V. vulnificus (1) & P. shigelloides (1) \\
\hline Exudado ótico (13) & $\begin{array}{l}\text { A. caviae (4) } \\
\text { A. hydrophila (2) } \\
\text { Aeromonas sp (1) } \\
\text { A. veronii bv sobria (1) }\end{array}$ & $\begin{array}{l}\text { V. cholerae no- } 01 \text { (2) } \\
\text { V. fluvialis (1) } \\
\text { V. cincinnatiensis (1) } \\
\text { V. alginolyticus (1) }\end{array}$ & \\
\hline Pus de herida (8) & $\begin{array}{l}\text { A. veronii bv sobria (2) } \\
\text { A. caviae (2) } \\
\text { A. trota (1) } \\
\text { A. veronii bv veronii (1) } \\
\text { Aeromonas sp (1) }\end{array}$ & V. mimicus (1) & \\
\hline Exudado conjuntival (5) & $\begin{array}{l}\text { A. caviae (2) } \\
\text { A. hydrophila (1) } \\
\text { A. veronii bv sobria (1) } \\
\text { A. schubertii (1) }\end{array}$ & & \\
\hline Urocultivo (5) & $\begin{array}{l}\text { A. hydrophila (2) } \\
\text { A. trota (1) } \\
\text { A. caviae (1) } \\
\text { Aeromonas sp (1) }\end{array}$ & & \\
\hline Catéter (4) & $\begin{array}{l}\text { A. caviae (2) } \\
\text { A. veronii bv veronii (1) } \\
\text { A. veronii bv sobria (1) }\end{array}$ & & \\
\hline Exudado lesión de piel (2) & A. hydrophila (2) & & \\
\hline Exudado faríngeo (2) & $\begin{array}{l}\text { A. caviae (1) } \\
\text { Aeromonas sp (1) }\end{array}$ & & \\
\hline Líquido cefalorraquídeo (1) & & & P. shigelloides (1) \\
\hline Bilicultivo (1) & & & P. shigelloides (1) \\
\hline Exudado secreción articular (1) & A. veronii bv sobria (1) & & \\
\hline Restos placentario (1) & A. caviae (1) & & \\
\hline Expectoración (1) & A. caviae (1) & & \\
\hline Exudado herida quirúrgica (1) & A. veronii bv sobria (1) & & \\
\hline Exudado úlcera de pie (1) & A. jandaei (1) & & \\
\hline Exudado endocervical (1) & A. caviae (1) & & \\
\hline Exudado vaginal (1) & A. caviae (1) & & \\
\hline Total & 85 & 7 & 3 \\
\hline
\end{tabular}

perados de muestras de animales, de ambientes acuáticos y del ser humano. En este último, dichos agentes están implicados como causa de gastroenteritis aguda, tanto en niños como en adultos, así como en la diarrea del viajero, particularmente en regiones tropicales y subtropicales. Desde hace varios años, estos microorganismos quedaron incluidos en la lista de agentes que producen infecciones extra-intestinales en pacientes inmunocompetentes e inmunocomprometidos $^{12}$.
La baja incidencia de aislamiento e identificación de estos microorganismos puede deberse a un inadecuado diagnóstico microbiológico, por la no sistematización de la prueba de oxidasa en los bacilos gramnegativos, anaerobios facultativos, procedentes de muestra extra-intestinales; en ocasiones, estas bacterias son clasificadas en géneros diferentes a los referidos anteriormente, entre los que se pueden mencionar Pseudomonas y Serratia o más aún, pueden ser confundidas con microorganismos contaminantes ${ }^{13}$. 
La mayor cantidad de los bacilos gramnegativos, oxidasa positiva, investigados a partir de las muestras extra-intestinales que formaron parte de nuestro estudio, pertenecieron al género Aeromonas. Nuestros resultados en relación a este género coinciden con lo publicado por Bravo L y cols, y con Pérez Peña S y cols, quienes en estudios realizados en Cuba en muestras extra-intestinales demostraron la mayor circulación del género Aeromonas ${ }^{14,15}$.

En el presente estudio las cepas procedían de múltiples orígenes clínicos, siendo los más frecuentes hemocultivos, exudados óticos, pus de heridas, exudados conjuntivales, urocultivos y catéter. Similares resultados han sido publicados por Brenden RA y cols, Howard RJ y cols y por Borrell $\mathrm{N}$ y cols, quienes en sus investigaciones demostraron el amplio espectro de circulación de estos microorganismos en muestras clínicas extra-intestinales ${ }^{16-18}$.

La diferenciación individual de las cepas, a nivel de especie, de los géneros que formaron parte de nuestro estudio, es de suma importancia clínica y epidemiológica, debido a la variación inter-especie, a sus capacidades invasoras y enterotoxigénicas, a los perfiles de susceptibilidad antimicrobiana y la distribución geográfica de cada especie ${ }^{19}$.

En este trabajo las cepas del género Aeromonas procedentes de las muestras de hemocultivos presentaron diversidad de especies y fueron aisladas de paciente con sepsis generalizada, ingresados en una unidad de cuidados intensivos; 10 cepas formaron parte de un brote de infección intrahospitalaria. Cuando se revisa la literatura científica internacional, nuestros resultados están acordes con lo publicado por Martino $\mathrm{R}$ y cols, los que hacen referencia a la identificación de diferentes especies de Aeromonas en muestras de hemocultivos ${ }^{20}$.

Vibrio vulnificus ha sido encontrado con anterioridad en E.U.A. por Dowdy LM y cols, en muestras de sangre de un paciente que recibió un trasplante de hígado después de ingerir ostiones crudos, y en Japón, por Osawa M y cols, quienes aislaron esta especie en un paciente con cirrosis hepática causada por el virus de la hepatitis $\mathrm{C}^{21,22}$. Hasta el presente no existían publicaciones de este género en muestras de hemocultivos en la literatura médica cubana.

Plesiomonas shigelloides, está asociada con alto grado de morbilidad y mortalidad en infección extraintestinal $^{23}$. Nuestros resultados están acordes con lo publicado por Riley PA y cols, quienes aislaron este microorganismo de un niño con leucemia, en una muestra de hemocultivos ${ }^{24}$.

Cuando se revisa la literatura científica especializada, son escasos los artículos publicados de aislados de Aeromonas en exudados de oído, no así para el género Vibrio. Brooks DE y cols, identificaron $A$. hydrophila en secreción de oído ${ }^{25}$. Otros autores como Mukherji A y cols, identificaron Vibrio alginolyticus en secreción de oído en un paciente que se expuso al agua salada ${ }^{26}$. Los resultados de nuestro estudio coinciden a los de los autores antes mencionados.

Revisando la literatura médica nacional e internacional pudimos constatar que no existen publicaciones acerca de $V$. fluvialis como causa de otitis; en este sentido, nuestros resultados ponen en evidencia la importancia de la búsqueda de este agente como causa de infección ótica ${ }^{27}$.

Las infecciones de heridas son la segunda fuente de aislamiento de Aeromonas y Vibrio, adquiriéndose por contacto con agua o suelo contaminado con estos microorganismos. Las mismas pueden tener un curso clínico leve o grave como tendinitis, necrosis muscular $\mathrm{y}$ osteomielitis, que en ocasiones dejan secuelas físicas permanentes para el individuo ${ }^{28}$.

Investigaciones clínicas realizadas por Bauab TM y cols, en Brasil, evidenciaron la presencia de microorganismos del género Aeromonas en muestras de pus de herida $^{29}$. De igual forma, Goodell KH y cols, aislaron Vibrio damsela en E.U.A., en un paciente que desarrolló una fascitis necrosante ${ }^{30}$. Los resultados expuestos en el presente trabajo fueron semejantes a los de los autores antes mencionados.

Filler G y cols, identificaron Aeromonas caviae en muestras de urocultivos ${ }^{31}$, hallazgo que concuerda con lo encontrado en nuestra experiencia.

La literatura científica recoge la escasa presencia de microorganismos pertenecientes a los géneros Aeromonas, Vibrio y Plesiomonas en muestras extra-intestinales tales como LCR, esputo, bilicultivo, secreción vaginal, exudado faríngeo, úlcera de pie, entre otras, como lo demuestra el trabajo publicado por Kao HT y cols $^{32}$.

El presente estudio demostró la amplia circulación y patogenicidad de estos agentes en diferentes especímenes clínicas. Se evidenció que con la aplicación sistemática de la prueba de oxidasa en los bacilos gramnegativos, anaerobios facultativos, oxidasa positiva, y la aplicación de los esquemas para la diferenciación en especie, fue posible contribuir a la mejor etiología de los procesos infecciosos producidos por los géneros Aeromonas, Vibrio y Plesiomonas en Cuba.

\section{Resumen}

Las infecciones extra-intestinales producidas por los géneros Aeromonas, Vibrio y Plesiomonas presentan una elevada tasa de morbimortalidad en diferentes áreas geográficas. De enero 2002 a diciembre 
2003 se recibieron en el Laboratorio Nacional de Referencia de Enfermedades Diarreicas Agudas del Instituto de Medicina Tropical Pedro Kourí, 95 cepas de bacilos gramnegativos, anaerobios facultativos, oxidasa positiva, procedentes de muestras extra-intestinales (hemocultivos, exudados óticos, pus de heridas, exudados conjuntivales, urocultivos, catéter, entre otras) remitidas de diferentes provincias del país. Se demostró la presencia de Aeromonas caviae, Aeromonas veronii bv sobria, Aeromonas jandaei, Vibrio cholerae no -01, Vibrio vulnificus, Vibrio fluvialis y Plesiomonas shigelloides en las muestras estudiadas.

\section{Referencias}

1.- Garrity G M, Bell J A, Lilburm T G. Taxonomic outline of the prokaryotes. En: Bergey S Manual of Systematic Bacteriology. 2nd ed. Release 4.0; Oct 2003. [Citado el 10 de febrero del 2004]. Disponible en: URL:http://dx.doi.org/10.1007/ bergeysoutline 200310

2.- Santamaría Muñoz R, Ramírez Aguilera P, Pansza R, Acevedo E, Hernández Estrada E. Sepsis por Vibrio cholerae en recién nacido. An Esp Pediatr 2002; 57: 361-3.

3.- Clark N M, Chenoweth C E. Aeromonas infection of the hepatobiliary system: Report of 15 cases and review of the literature. Clin Infect Dis 2003; 37: 506-13.

4.- Woo P C, Lau S K, Wong S S, Yuen K Y. Two cases of continuous ambulatory peritoneal dialysis-associated peritonitis due to Plesiomonas shigelloides. J Clin Microbiol 2004; 42: 933-5.

5.- Ghenghesh K S, Bara F, Bukris B, El-Surmani A, Salaheddin S A. Characterization of virulence factors of Aeromonas isolated from children with and without diarrhoea in Tripoli, Lybia. J Diarrhoeal Dis Res 1999; 17: 75-80.

6.- Atkins J T, Cleary T G. Aeromonas and Plesiomonas. En: Behrman $\mathrm{H}$, editor. Nelson Texbook of Pediatrics. 16 ed. España: Mc Graw -Hill-Interamericana; 2000. p. 861-3.

7.- Chin J. El control de las enfermedades transmisibles. 17 ed. Washington, DC.: OPS: 2001. Publicación científica y técnica No. 581.

8.- Carnahan A M, Behran S, Joseph S W. Aerokey II: a flexible key for identifying clinical Aeromonas species. J Clin Microbiol 1991; 29: 2843-9.

9.- Furuwatari C H, Kawakami Y, Akahame T, Hidaka E, Okimura Y, Nakayama J, et al. Proposal for an Aeroscheme (modified Aerokey 11) for the identification of clinical Aeromonas species. Med Sci Res 1994; 22: 617-9.

10.- Charles A, Angelo DePaola J.

Bacteriological analytical manual on line. May 2004. Chapter 9. 8th ed. [citado el 15 de mayo del 2004]. Disponible en URL: http://vm.cfsan.fda.gov/ ebam/bam-9.html

11.- Janda J M, Powes C, Bryant R G, Abbott S L.
Current perspective on the epidemiology and pathogenesis of clinically significant Vibrio spp. Clin Microbiol Rev 1988; 1: 245-67.

12.- Stock I, Wiedemann B. Natural antimicrobial susceptibilities of Plesiomonas shigelloides strains. J Antimicrob Chemother 2001; 48: 803-11.

13.- Melhus A, Holmdahl T, Tjernberg I. First docummented case of bacteremia with Vibrio vulnificus in Sweden. Scand J Infect Dis 1995; 27: 81-2.

14.- Bravo Fariñas L, Monte R J, Gómez M, Dumas S C. Identificación de bacilos Gramnegativos, anaerobios facultativos, oxidasa positivos. Rev Cubana Med Trop 1993; 45: 46-8.

15.- Pérez Peña S, Tamayo Curro E, Díaz García M E, Pérez Rodríguez J M. Primer reporte en Cuba de aislamiento de Aeromonas en muestras extraintestinales. Rev Cubana Med Milit 1997; 26: 50-4.

16.- Brenden R A, Miller M A, Janda J M. Clinical disease spectrum and pathogenic factors associated with Plesiomonas shigelloides infections in humans. Rev Infect Dis 1988; 10: 303-16.

17.- Howard R J, Bennett N T. Infections caused by halophilic marine Vibrio bacteria. Ann Surg 1993; 217: 525-30.

18.- Borrel N, Figueras M J, Guarro J. Phenotypic identification of Aeromonas genomospecies from clinical and environmental sources. Can J Microbiol 1998; 44: 103-8.

19.- Hsueh P R, Teng L J, Lee L N, Yang P C, Chen Y C, Ho S W, et al. Indwelling devicerelated and recurrent infections due to Aeromonas species. Clin Infect Dis 1998; 26: 651-8.

20.- Martino R, Gómez L, Pericas R, Salazar R, Sola C, Sierra J, et al. Bacteraemia caused by non-glucose-fermenting Gram-negative bacilli and Aeromonas species in patients with haematological malignancies and solid tumours. Eur J Microbiol Infect Dis 2000; 19: 320-3.

21.- Dowdy L M, Khan F, Regalado J, Tzakis A G. Primary Vibrio vulnificus bacteremia in a liver transplant recipient after ingestion of raw oysters: caveat emptor. Transplantation 1999; 68: 1061-3.

22.- Osawa M, Tachibana M, Arita M, Hashimoto T, Ishida T, Hongo T, et al. An autopsied case of septicemia due to Vibrio vulnificus. Kansenshogaku Zasshi. 2002; 76: 63-4.

23.- Bravo L, Cabrera R, Ramírez M, Llop A, Fernández A, Concepción A, et al. Fatal Plesiomonas shigelloides in newborn. Mem Inst Oswaldo Cruz 1999; 94: 661-2.

24.- Riley P A, Parasakthi N, Abdullah W. Plesiomonas shigelloides bacteremia in a child with leukemia. Clin Infect Dis 1996; 23: 206-7.

25.- Brooks D E, Jacobson E R, Wolf E D, Clubb S, Gaskin J M. Panophthalmitis and otitis interna in fire-bellied toads. J Am Vet Med Assoc 1983; 183: 1198-201.

26.- Mukherji A, Schroeder S, Deyling C, Procop G W. An unusual source of Vibrio alginolyticus-associated otitis: prolonged colonization or freshwater exposure? Arch Otolaryngol Head Neck Surg 2000; 126: 790-1.

27.- Cabrera Rodríguez L E, Palma Monroy S, Ramírez Álvarez M, Castro Escarpulli G, Cabrera Ortega R, Bravo Fariñas L. Severe otitis due to Vibrio fluvialis in a patient with AIDS: first report in the world. Rev Cubana Med Trop 2005; 57: 154-5.

28.- Varghese M R, Farr R W, Wax M K, Chafin B J, Owens R M. Vibrio fluvialis wound infection associated with medical leech therapy. Clin Infect Dis 1996; 22: 709-10.

29.- Bauab T M, Levy C E, Rodríguez J, Pasetto Falcao D. Niche-specific association of Aeromonas ribotypes from human and environmental origen. Microbiol Immunol 2003; 47: 7-16.

30.- Goodell K H, Jordan M R, Graham R, Cassidy C, Nasraway S A. Rapidly advancing necrotizing fasciitis caused by Photobacterium (Vibrio) damsela: a hyperaggressive variant. Crit Care Med 2004; 32: 278-81.

31.- Filler G, Ehrich J H H, Lothar E S B. Acute renal failure in an infant associated with cytotoxic Aeromonas sobria isolated from patient's stool and from aquarium water as suspected source of infection. J Clin Microbiol 2000; 38: 469-70.

32.- Kao H T, Huang Y C, Lin T Y. Fatal bacteremic pneumonia caused by Aeromonas hydrophila in a previously healthy child. J Microbiol Immunol Infect 2003; 36: 209-11. 